\title{
Community Power Flow Control for Peak Demand Reduction and Energy Cost Savings
}

\author{
Seksak Pholboon, Mark Sumner and Petros Kounnos \\ Department of Electrical and Electronic Engineering, \\ University of Nottingham, Nottingham, UK \\ seksak.pholboon@nottingham.ac.uk
}

\begin{abstract}
The increase in penetration of renewable energy sources, such as solar or wind, and high peak load demand can cause grid network security issues. The incorporation of demand side management and energy storage devices can provide a solution to these problems. This paper presents a community power flow control (PFC) strategy which reduces peak grid demand, and increases self-consumption of renewable energy which produces energy cost savings in smart communities with grid-connected photovoltaic (PV) systems. The PFC aims to directly control high power consumption appliances and the charge/discharge of a community battery storage using measurement of the instantaneous power demands of the community. Historical data records of the community daily energy consumption and the available renewable energy are taken into account to manage the loads and battery storage. Simulation results show for a community of one hundred houses, with $114 \mathrm{kWp}$ of PV arrays, and a 350kWh battery system that the percentage of the average peak power demand reduction over the year is $32 \%$, whilethePV energy selfconsumption increases by $73 \%$. This can produce an annual energy cost saving of up to $£ 1100$ when compared to the same community with only PV.
\end{abstract}

Index Terms--Battery energy storage, demand side management, peak demand reduction, power flow control, PV system,

\section{INTRODUCTION}

World electricity demand almost doubled from 1990 to 2011 and seems set to grow further by $81 \%$ from 2011 to 2035 (from $19,004 \mathrm{TWh}$ to $34,454 \mathrm{TWh}$ ) [1].In order to respond to this growth, an natural gas power plant may be favorable, which could result in a rise in electricity price, and greenhouse gas emissions. To cope with these issues, sustainable energy sources such as solar power, wind power and biomass energy are receiving increased attention. In the UK, the government has been encouraging households to install PV panels by offering Feed-in Tariffs. This has resulted in significant growth of PV installations at a domestic level from a cumulative $7 \mathrm{MWp}$ in 2010 to over 2,373 MW in mid of 2016 [2]. However, these intermittent renewable energy sources do not always help to reduce the peak demand at times of consumption and generation mismatch. Furthermore, in some locations, grid problems have been observed where a high level of PV generation is injected into the distribution system during days with strong solar irradiance, and low load demand. This can cause serious issues to the distribution system such as high system losses, voltage regulation and electricity blackout [4],[5]. The grid infrastructure may also need to be upgraded in order to cope with these power flows and hence substantial investment could be required in certain locations. One solution is to deploy a combination of demand side management (DSM) and energy storage (ES).The DSM provides the opportunity for load shifting from the peak to off-peak periods and also the possibility to control the turn on of loads during high PV generation. At the same time, the ES stores energy from the excess generated by PV or from importing from the grid during times of cheap electricity price. This stored energy can then be used during periods of high demand [3] thereby reducing the peak.

In order to maximize the benefit of the system, the control approach needs to be focused. [6],[7] presented the power flow control approach which regulated instantaneous power and energy consumption of energy communities ie groups of electricity users who worked cooperatively. The advantage of working as communities is that this is more likely to allow access to a time of use tariff (certainly in the near future) and also can benefit from economies of scale for ES and control equipment, and also it will increase community selfconsumption. The Power Flow Control approach sets the power target set-point based on the predicted average community power demand. Then the instantaneous community power is compared to the power target to make the decision whether the ES should be charged or discharged and the loads should be switched on or delayed. However, the problems of the constant power flow target are that the energy stored within ES is less than the energy required during the peak demand due to high demand variation and also there is little benefit in terms of the energy cost savings when considering the real-time energy price where the energy price varies every half-hour. This paper presents a proposed power flow control strategy which uses a variable power target which eliminates the weakness found in [6],[7]. The major benefits of the incorporation of DSM and battery storage systems are to shave the peak power demand and to increase renewable self-consumption, resulting in increased grid stability and reduced distribution system losses. There are two potential energy tariff programs: time-of-use (ToU) and real-time price (RTP) which can provide consumers the benefit of reducing their energy bill by deploying this system [8]. In this work, the RTP based directly on the wholesale market price is considered.

The paper is organized as follows: Section II presents the community power flow control (CPFC) strategy. Section III discusses the data sources used for the case study including the power converter used as an interface and battery system model 
obtained from an experiment. Section IV presents the outputs of simulations that illustrate the results of the system operation; peak demand reduction, PV self-consumption and energy cost savings. Finally, Section V presents conclusions drawn out of this work.

\section{COMMUNITY POWER FLOW CONTROL}

The CPFC aims to reduce the peak community demand, maximize renewable self-consumption and minimize energy bills by controlling DSM based direct load control and the battery storage. The controller selects the target power set-point based on average power demand for the community (the socalled Community Power Target $(C P T)$ ) and then connects or shifts the load and may charge or discharge the battery whenever demand is below or above the $C P T$. In this case, the demand is always equal to the average as long as the $C P T$ is close to or slightly higher than the actual average demand and the battery capacity is large enough. However, in terms of the energy cost savings due to the fluctuation of the RTP every half hour, this algorithm is not the best. When considering the energy price, the $C P T$ must be modified to optimize for both the cost and the peak demand. The approach is to increase the $C P T$ to allow the load to be switched on or increase stored energy in the battery during low price periods and decrease the $C P T$ to avoid the load being switched on or discharge the battery to reduce community energy demand during the high price periods. However, to prevent the battery from charging at too high a power, leading to a large low-price peak demand, we use the ratio between the RTP and the mean price to determine the level of how much the $C P T$ needs to vary around the average power. Furthermore, a minimum $C P T$ limit needs to be set in the case of too high a RTP to avoid the battery being over discharged, and ensure that there will be enough energy for the evening peak. Hence, the CPT can be expressed as (1):

$$
C P T= \begin{cases}\frac{-\left(E_{R T P}-\bar{E}_{R T P}\right)}{\bar{E}_{R T P}} \times \bar{P}_{d m_{-} P D}+\bar{P}_{d m_{-} P D}, E_{R T P}<2 \cdot \bar{E}_{R T P} \\ P_{d m_{-} \text {base }} & , E_{R T P} \geq 2 \cdot \bar{E}_{R T P}\end{cases}
$$

where $E_{R T P}$ is a real-time price in $£ / \mathrm{kWh}$ and $\bar{E}_{R T P}$ is the mean price per kWh. In addition, $P_{d m_{-} \text {base }}$ is a base demand in $\mathrm{kW}$ and $\bar{P}_{d m_{-} P D}$ is a prediction of the mean demand in $\mathrm{kW}$ if all the locally-generated renewable energy can be captured and used within the community, which can be given as (2):

$$
\bar{P}_{d m_{-} P D}=\frac{\left(E_{C}-E_{R E S}\right)}{24}
$$

where $E_{C}$ is a prediction of the community energy consumed for the next day in $\mathrm{kWh}$ and $E_{R E S}$ is a prediction of the local renewable energy available in $\mathrm{kWh}$ over the next 24 hours. The weather conditions for the next day can be accessed from weather forecasting websites and this can help predict the total renewable energy available for the next day. It is assumed that as research progresses in this field a sufficiently accurate prediction one day ahead will be available in the future, certainly for PV systems. The load profile as an hour-by-hour or minuteby-minute prediction is more difficult to forecast accurately. However, the whole day's energy demand can be estimated using energy demand of the equivalent day of the previous week and with this approach complicated methods for prediction are not required [9],[10].

\section{A. Real-Time Control}

As mentioned earlier, the community power flow controller operates by comparing the actual instantaneous community power $(C P)$ and with the $C P T$. The summary of the algorithm is described below:

- If the $C P$ is below the $C P T$ then, check if there are any appliance requests or any waiting to be switched on. If yes, check how many can be allowed so that the $C P$ for the next sampling time will not go above the $C P T$. After the decision to turn on appliances, a check whether there is enough power to charge the battery or not is made. If there are no request signals from the appliances, then charge the battery at the power equal to the power difference between $C P$ and $C P T$.

- If the $C P$ is above the $C P T$ then, delay the request appliance signal and also discharge the battery

There are three main controllable appliances considered: washing machine, tumble dryer, and dishwasher. The maximum time delay for each appliance is set at two hours for consumer convenience. At any moment, all appliances are allowed to run to complete their full cycle due to the physical nature of the equipment. This is unlike some other possible equipment/appliances such as fridges, hot water tanks or electric space heaters that can be stopped as required.

\section{DATA SOURCES AND MODELING}

\section{A. Residential Community Model}

A simulation of domestic demand was created using a model from the Centre for Renewable Energy Systems Technology (CREST) created by Richardson and Thompson[9]. This CREST tool was used to generate 100 residential power profiles with a time resolution of one minute including the on/off switch signals for individual appliances for each house. These on/off appliance signals were used as a request signal fed into the DSM controller for it to decide whether that appliance is allowed to turn on at the requested time period. There are three high power appliances: washing machine, tumble dryer and dishwasher which are considered as controllable loads.

\section{B. Renewable Energy Sources}

For the PV generation, it is assumed that $30 \%$ of consumers install a $3.8 \mathrm{~kW}$ (peak) PV array for local generation. This equates to a PV penetration level as high as $100 \%$ of the total community peak demand power. The input for the simulation is obtained from data recorded at 10-minute intervals made publicly available on www.pvoutput.org and the location of the PV data is in Nottingham, UK.

\section{Energy Prices}

The economic case for adding a direct load control system and battery storage to a smart energy community cannot be made using fixed energy price rate where the energy is charged per $\mathrm{kWh}$ at a constant price. There needs to be a significant price differential between peak and off-peak consumption periods to help drive value creation for DSM and battery systems in typical residential dwellings. There are two types of time variable 
energy price schemes; Time of Use (ToU) and Real-Time Price (RTP). The ToU provides two or three price levels so called 'off-peak', 'mid-peak' and 'peak' while the RTP is based on hourly or half-hourly price differences to reflect the price on the wholesale market [12]. The study in [13] concludes that the RTP delivers the most benefits in terms of reducing the peak and flattening the demand. Therefore, the RTP based directly on the wholesale market has been considered in this work. The dataset used for the energy price of the RTP, is based on the total UK electricity consumption over the year of 2011 and can be found from New Electricity Trading Arrangements (NETA) [14]. The data shows the energy price per MWh divided into half hour blocks and also contains both selling and buying prices which can be used to calculate the energy cost when the energy has been imported (load demand and charging battery) or exported (excess PV generation).

\section{Power Converter Model}

The power converter model in this work is obtained from the experiment in the laboratory at the University of Nottingham [15]. The available $12 \mathrm{~kW}$ power converter has been tested across a range of operating power and the input and output powers have been measured. As a result, the output power equation of this converter derived from measured dataset can be expressed as (3).

$$
P_{\text {out }}= \begin{cases}0.95 * P_{\text {in }}-323.89, & P_{\text {in }}>0 \\ 0 & , P_{\text {in }}=0\end{cases}
$$

where $P_{\text {out }}$ is the output power of the converter and $P_{\text {in }}$ is the input power in watts. It can be found from (3) that the efficiency $(\eta)$ of the converter becomes extremely poor as the operating power reduces. This would affect the total energy cost saving corresponding to the payback of the system when the charge/discharge decision process was made. This power converter can also be scaled to match with the battery converter interface power requirement, which will be used for the simulation case study.

\section{E. Battery Model}

The parameters that determine usage of the battery are the instantaneous battery voltage (battery terminal voltage) and battery degradation. The battery voltage evolves with the battery state of charge and battery degradation is associated with capacity fade and resistance rise. These parameters will determine the maximum charge- and discharge- power of the battery as well as the maximum battery capacity. In order to determine the instantaneous battery voltage, a simple battery equivalent circuit is considered. Two important battery parameters are the battery open-circuit voltage and battery internal series resistance which can be derived from the experiments of previous work [15].In terms of the battery degradation, the battery manufacturer data sheet provides the curves of cycle life and calendar life. The degradation of the internal resistance information of the battery cell has been derived from the SAFT battery pack [16].

\section{CASE Study AND Simulation Results}

The proposed PFC is verified through simulations using MATLAB software. The case study is based on 100residential homes linked as an energy community. It is also assumed that there are thirty houses installing 3.8kWp PV array, which make up the total PV peak power of $114 \mathrm{~kW}$. The community energy storage deploys a $50 \mathrm{~kW} 350 \mathrm{kWh}$ lithium-ion battery system where its power rated and capacity have been selected based on the optimal power and energy usage for the battery each day throughout the year. These can be worked out by running the CPT and the CP profiles together and considering the differential power and energy between CPT and CP, which indicates the amount of power and energy required to charge (when CPT level is greater than the $\mathrm{CP}$ ) or discharge (when CPT level is lower than the $\mathrm{CP}$ ) the battery. By applying the probability density function method on differential power and energy, the most likely operating range of battery power and capacity are defined. In terms of the load control consideration, each house is assumed to have installed remote control switches at all three controllable appliances to give a total of 300 appliances to be controlled. It is considered that the load shifting may be undesirable for a consumer and therefore the consumers are allowed to override the control switch if preferred. It is assumed that only $20 \%$ of all appliances are compliant to the DSM controller each day. There are two main conditions regarding the load shifting strategy. One is the load can be delayed to a maximum period of two hours. The other is if the load is delayed after $20.00 \mathrm{hrs}$, that load will be shifted further and started from $04.00 \mathrm{hrs}$ on the next day. To extend the battery life time, the battery state of charge is limited to be between $10 \%$ and $90 \%$. The simulations compare the algorithms between the RTP driven CPT (RTPDCPT) and the CPT based average power (CPTBAVP).

The results of operating the DSM and battery system are given in the various plots of Figure 1showing a typical winter day. Figure1(a) shows the underlying community power demand (red), and the community demand when including the PV generation and DSM without (black) and with (blue) the battery. The $C P T$ is shown in green for reference and the PV generation is shown in yellow. Figure 1(b) is similar to Figure 1(a) but shows a system with the CPTBAVP. Figure 1(c) shows the RTP in $f / k W h$. Figure 1 (d) shows the battery state of charge. Similar graphs for summer will also be presented in Figure 2.

It can be seen in Figure 1 that in winter, with less PV generation, for the RTPDCPT algorithm the battery charges more energy than the CPTBAVP overnight. This causes the battery with the CPTBAVP to run out too early at $17.00 \mathrm{hrs}$ resulting in a large amount of peak energy consumption left during the evening high price period, whilst the battery with the RTPDCPT can be utilized throughout the evening peak period. The DSM controller also helps the battery to shift the load as seen from the comparison between the black and red lines. Between $04.00 \mathrm{hrs}$ and $05.00 \mathrm{hrs}$, the demand on black line is obviously higher than that on the red. This is due to the appliances having been shifted from the previous day evening high price periods. Furthermore, between $16.00 \mathrm{hrs}$ and $18.00 \mathrm{hrs}$ when the RTP reaches its peak, the black line runs below the red line. This again shows that the DSM is able to shift the load away from the peak. In summer, as shown inFigure2, the CPT is set low due to expected high PV generation during the daytime 
and therefore the battery charges a small amount of energy overnight which can be utilized during the early morning. Then the battery starts to charge at a high rate using the excess PV energy until it is full (at $90 \%$ SOC) when the PV generation nearly ends for the day. Both algorithms are able to flatten the demand through the whole day. However, the RTPDCPT has an advantage by making use of the energy price difference to reduce energy cost further.
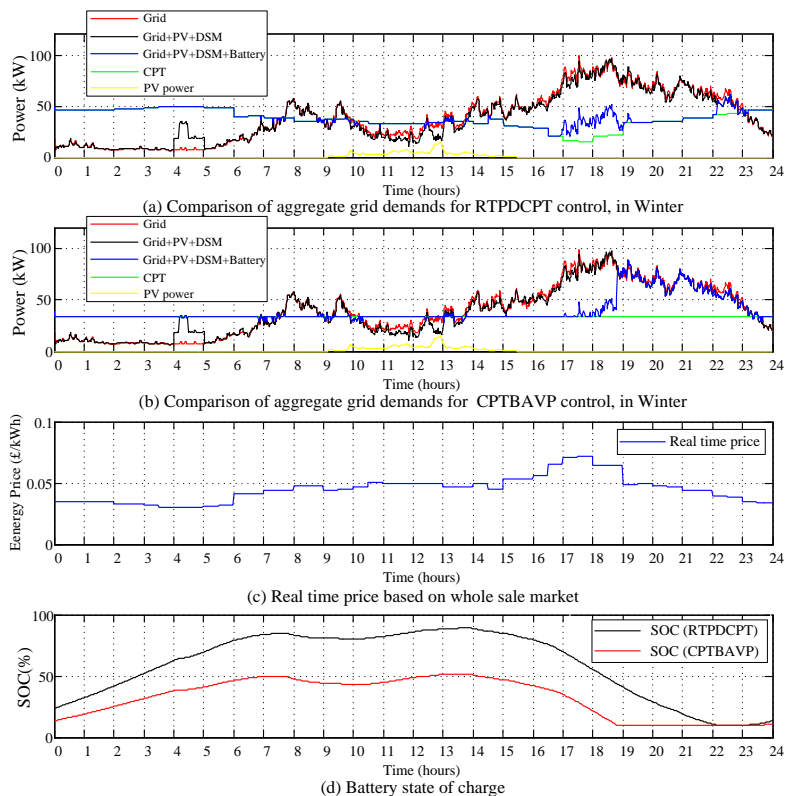

Figure 1Simulation Results in Winter: (a) Community Grid Demand for the RTPDCPT control, (b) Community Grid Demand with CPTBAVP control (c) Real-time price, and (d) Battery State of Charge.

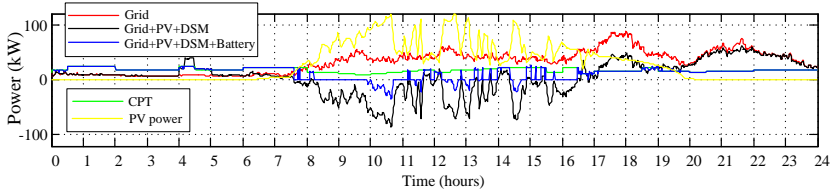

(a) Comparison of aggregate grid demands for RTPDCPT control, in Summer

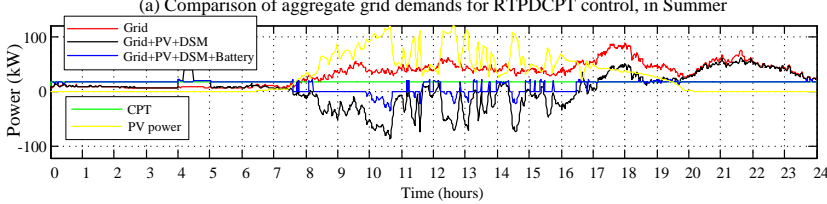

(b) Comparison of aggregate grid demands for CPTBAVP control, in Summer

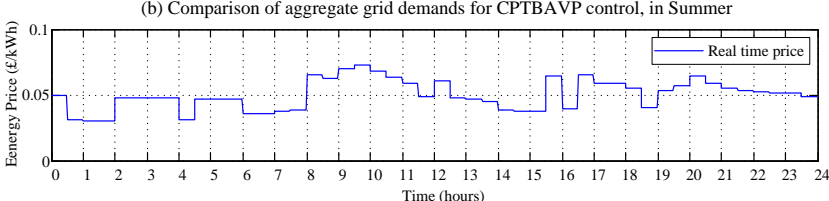

(c) Real time price based on whole sale market

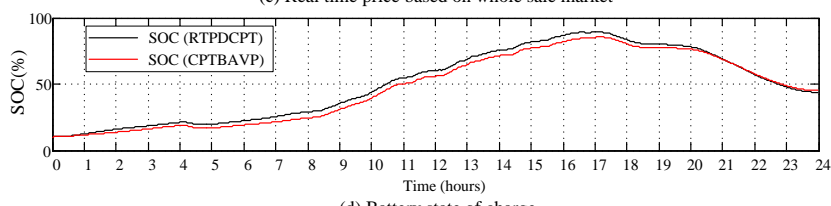

(d) Battery state of charge

Figure 2Simulation Results in Summer: (a) Community Grid Demand for the RTPDCPT control, (b) Community Grid Demand with CPTBAVP control (c) Real-time price, and (d) Battery State of Charge.
The overall performance of the algorithm can be quantified by assessing how the DSM and battery change energy consumption patterns - particularly its ability to move consumption from high to low price periods. In order to make it simple, four different energy consumption zones have been categorized. An expensive zone (high price periods) is identified where the energy prices are above $10 \%$ of the average price. A cheap zone is located where the energy prices are below $10 \%$ of the average price whilst all other are assumed to be an average zone. An export zone is considered to be where the net demand is below zero, the excess generated PV energy is exported to the grid. Figure 3 shows the total community energy consumption over the year for the five scenarios considered. Due to the high PV penetration, the grid with PV array (blue) can reduce the energy consumption from each zone by approximately $30 \%$ compared to grid supply only (red). By adding DSM and battery (green) for RTPDCPT control the expensive-rate consumption is moved to the cheap-rate increasing to around $30 \%$ of total community demand whilst $10 \%$ less for the CPTBAVP control. The DSM and battery also considerably reduces the amount of PV energy exported, by $73 \%$ (i.e. self-consumption is significantly increased).

In order to quantify the benefit of peak power demand reduction, Figure 4 shows the average percentage of the peak demand reduction over the year. It is obvious that the DSM and battery provides a significant peak reduction close to $20 \%$ even though there is no PV array installed and about $10 \%$ more with the PV array.

The energy cost saving based on the actual wholesale market prices is shown in Figure 5. It is clear that the RTPDCPT control delivers a great energy cost saving by more than double compared to the CPTBAVP control. However, the saving is incomparable to the capital cost of the DSM and battery system because the energy prices are currently still low and also this analysis does not include the cost of peak demand charge based on the highest peak power demand in $\mathrm{kW}$ which is charged by the distribution network and transmission network operators.

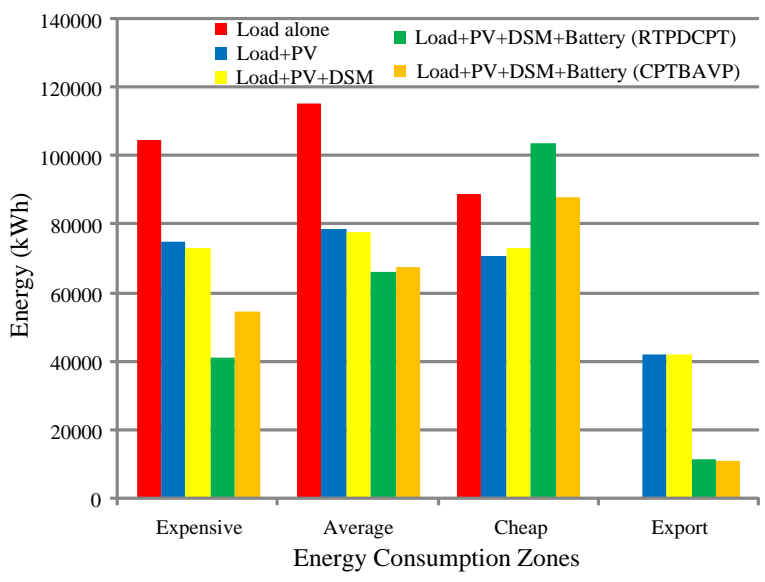

Figure 3Annual Community Energy Consumption at Different Price Zones 


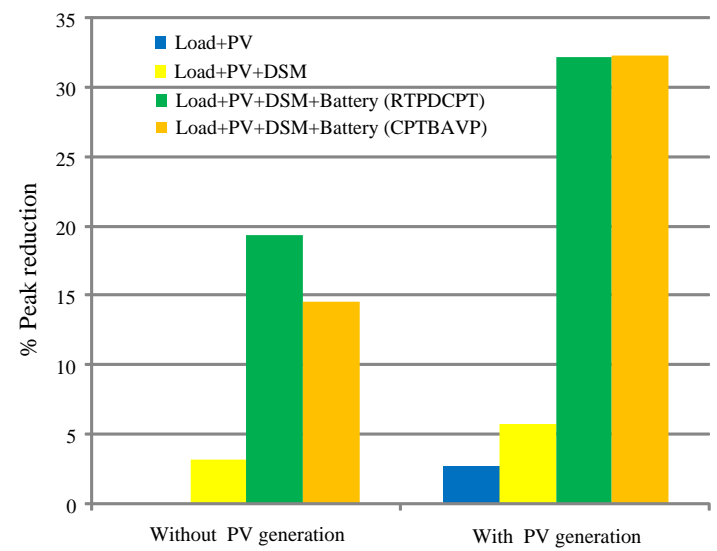

Figure 4Average Percentage of the Peak Demand Reduction over the Year.

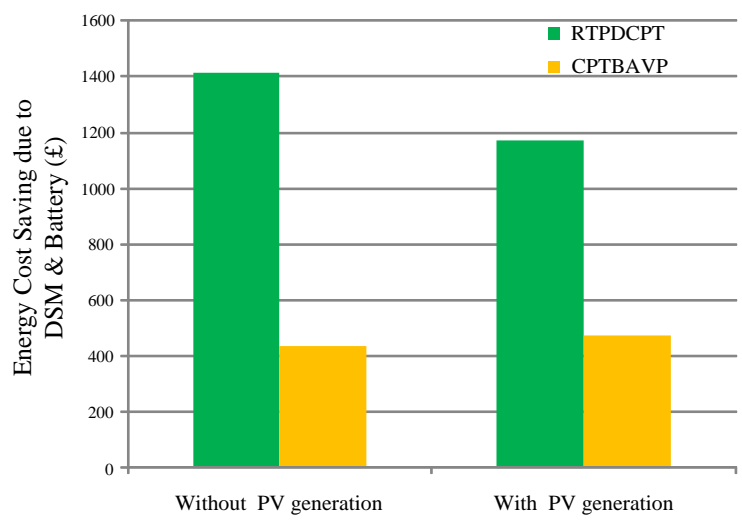

Figure 5Energy Cost Saving over the Year.

\section{CONCLUSIONS}

This paper develops a CPFC algorithm to reduce the peak energy and power demand, improve energy cost savings and increase renewable self-consumption through use of a DSM and community battery storage system. The proposed CPFC uses historical load (previous week, same day), PV data (previous day) and the RTP based directly on the wholesale energy market as input variables to determine the DSM control and the battery charge/discharge decision through creation of a CPT set point. Two algorithms which are RTPDCPT and CPTBAVP are compared to prove the proposed RTPDCPT can perform better in terms of reducing energy cost and peak demand while being more robust to error in predictions. The simulation study reveals that with CPTBAVP control the battery often runs out early before fully peak-shaving the evening peak period. This could be due to an error when predicting the average power demand. Since the CPT is based on the average power prediction, if the predicted average is below the actual average demand, then the battery will charge less and therefore there is not enough energy for the high price or peak periods. This scenario could happen to both RTPDCPT and CPTBAVP controls however; as the RTPDCPT also uses the RTP to vary the CPT from the average power. As a result, the battery can have more energy coming in during the low price when the CPT is increased. The effectiveness of the CPFC indicates that the average peak demand over the year can be reduced by $32 \%$ with an increase in the PV self- consumption by $73 \%$ as well as a reduction of $£ 1100$ energy cost when compared to a similar community with PV but without the DSM or battery storage.

\section{ACKNOWLEDGMENT}

This research was supported by Engineering and Physical Sciences Research Council, EPSRC, UK, grants EP /K036297/ 1, "Intelligent MicroGrids with Appropriate Storage for Energy Project (IMASE)".

\section{REFERENCES}

[1] The OECD's International Energy Agency, "The World Energy Outlook 2013,'Tech. Rep.[Online].Available: $\quad$ http://www.oecdilibrary.org/energy/world-energy-outlook-2013 weo-2013-en.

[2] Department of Energy and Climate Change, London, "Solar Photovoltaics Deployment," Tech. Rep., May 2016 [Online]. Available: https://www.gov.uk/government/statistics/solar-photovoltaicsdeployment

[3] M.J. Knowles, and A. Morris, "Impact of Second Life Electric Vehicle Batteries on the Viability of Renewable Energy Sources,"British Journal of Applied Science \& Technology, pp.152-164, 2014.

[4] Sandia National Laboratories, "Solar Energy Grid Integration Systems," Program Concept Paper, October 2007.

[5] J.D. Dogger, B. Roossien, and F.D.J. Nieuwenhout, "Characterization of Li-Ion Batteries for Intelligent Management of Distributed GridConnected Storage,"IEEE Tranl.Energy Conversion, Vol. 26, pp. 256263, 2011.

[6] A Fazeli, M. Sumner, C.M. Johnson, and E. Christopher, "Coordinated Optimal Dispatch of Distributed Energy Resources within a Smart Energy Community Cell,"'IEEE PES Innovative Smart Grid Technologies Europe Conf., pp.1-10, 2012.

[7] A Fazeli, M. Sumner, C.M. Johnson, and E. Christopher, "Investigating the Impact of Varying the Number of Distributed Energy,"IEEE PES Innovative Smart Grid Technologies Conf.,pp.1-5, 2015.

[8] A. Mishra, D. Irwin, P. Shenoy, and T. Zhu,"Scaling Distributed Energy Storage for Grid Peak Reduction," inProc.ACM e-Energy, Berkeley, USA,pp3-14, May 2013.

[9] N. Sharma, J. Gummeson, D. Irwin, and P. Shenoy, "Cloudy computing: Leveraging weather forecasts in energy harvesting sensor systems," in SECON, June 2010.

[10] N. Sharma, P. Sharma, D. Irwin, and P. Shenoy, "Predicting Solar Generation from Weather Forecasts Using Machine Learning," in SmartGridComm, October 2011

[11] I. Richardson,and M. Thomson, "Domestic electricity demand model simulation example," ed, 2010.

[12] P. Van De Ven, N. Hegde, L. MAssoulie, and T. Salonidis, "Optimal Control of Residential Energy Storage Under Price Fluctuations, "Smart Grids, Green Communications and IT Energy-aware Technologies, pp.159-162, 2011.

[13] S. Borenstein, M. Jake, and A. Rosenfeld, "Dynamic pricing, advanced metering, and demand response in electricity markets," UC Berkeley: Center for the Study of Energy Markets.

[14] Anonymous, "The New Electricity Trading Arrangements for the Imbalance Market in UK 2011," ELEXON Ltd, UK, [Online]. Available: http://www.bmreports.com/bsp/SystemPricesHistoric.htm/.

[15] S. Pholboon, M. Sumner, E. Christopher, and S.A. Norman, "Real-Time Battery Management Algorithm for Peak Demand Shaving in Small Energy Communities, "IEEE PES Innovative Smart Grid Technologies Latin America Conf., pp.19-24, 2015.

[16] E. Wood, M. Alexander, and T.H. Bradley, "Investigation of battery endof-life conditions for plug-in hybrid electric vehicles, "Power Sources.,pp.5147-5154, 2011. 